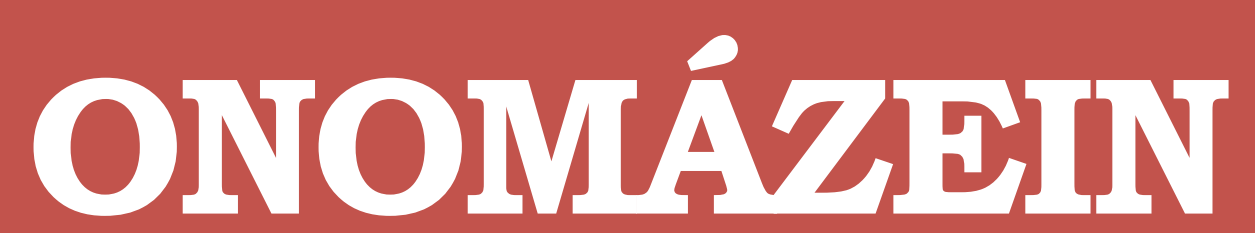

Revista de lingüística, filología y traducción
PONTIFICIA UNIVERSIDAD CATÓLICA DE CHILE FACULTAD DE LETRAS

\title{
Actos de habla expresivos en la red social Facebook ${ }^{1}$
}

Expressive speech acts in the social network Facebook

\section{Susana Ridao Rodrigo \\ Universidad de Almería \\ España}

\section{(C) $($ (i) $\ominus$}

Susana Ridao Rodrigo: Departamento de Filología, Facultad de Humanidades Universidad de Almería, España. | E-mail: sridao@ual.es 


\section{Resumen}

El objetivo de este artículo es analizar, desde el perfil cuantitativo y cualitativo, los subtipos de actos de habla expresivos utilizados por usuarios de la red social Facebook con perfiles privados. Tras indagar en la teoría de los actos de habla, se detallan las subtipologías de actos de habla expresivos diseñadas por Searle (1976), Norrick (1978), Carretero y otros (2014) y Maíz-Arévalo (2017). La metodología empleada en esta investigación ha sido creada ad hoc teniendo en cuenta, por un lado, las taxonomías ya existentes y, por otro lado, las necesidades específicas del corpus. Animar conforma una subtipología que no aparece en los modelos citados. Como resultado, se ha de destacar que felicitar es la opción más utilizada. He aquí los porcentajes: felicitar (45.19\%), agradecer (16.72\%), aprobar (9.96 \%), animar (8.54\%), admirar (7.11\%), saludar (6.76\%), dar la bienvenida (2.13\%), lamentar (1.77\%), transmitir alegría (1.42\%) y desaprobar (0.35\%).

Palabras clave: pragmática; actos de habla expresivos; redes sociales; Facebook.

\section{Abstract}

The aim of this paper is to analyze, from the quantitative and qualitative profile, the subtypes of expressive speech acts used by users of the social network Facebook with private profiles. After inquiring into the theory of speech acts, the subtypes of expressive speech acts designed by Searle (1976), Norrick (1978), Carretero and others (2014) and Maíz-Arévalo (2017) are detailed. The methodology used in this research has been created ad hoc taking into account, on the one hand, the previous taxonomies and, on the other hand, the specific needs of the corpus. Animating conforms a subtype that does not appear in the cited models. As a result, it should be noted that congratulating is the option most used. These are the percentages: congratulate (45.19\%), thank (16.72\%), approve (9.96\%), animate (8.54\%), admire (7.11\%), greet (6.76\%), welcome (2.13\%), lament (1.77\%), transmit joy (1.42\%) and disapprove (0.35\%).

Keywords: pragmatics; expressive speech acts; social networks; Facebook.

1 Este artículo nace al amparo del proyecto de investigación "Pragmática y gramática en la historia del español: la expresión de la cortesía en el español clásico" (FFI2014-53113-P), financiado por el Ministerio de Economía, Industria y Competitividad, y del grupo de investigación HUM783, el cual está vinculado al centro de investigación CEMyRI de la UAL. 


\section{Introducción}

"Internet es mucho más que una tecnología. Es un medio de comunicación, de interacción y de comunicación social", asevera el sociólogo Manuel Castells. Ciertamente, las innovaciones tecnológicas repercuten en la permuta de las formas de comunicarse, que a su vez afecta a la terminología, los signos, los símbolos o la lengua (Parrilla, 2008: 131-132), cambios que no han estado exentos del debate de si se trata de un proceso de evolución o de involución (Thurlow, 2014). Del mismo modo, no se puede olvidar que la comunicación en el contexto digital constituye un género híbrido que está a caballo entre el plano oral y el escrito, porque a menudo los mensajes escritos intentan reproducir rasgos orales (Ridao Rodrigo y Rodríguez Muñoz, 2013: 84). Además, las redes sociales — tan extendidas hoy en día - implican nuevas formas de comunicación que requieren de normas específicas, dado que en estos contextos convergen cuestiones propias de la comunicación presencial con aspectos de la esfera virtual (Yus, 2010: 141).

Las investigaciones lingüísticas enseguida se percataron de que la expansión de internet repercutía directamente en la comunicación entre los individuos, tal como vaticinó Crystal (2001). Señalan Giammatteo, Gubitosi y Parini (2017: 23) que el español es una de las lenguas que goza de mayor presencia en la red, de ahí el interés existente en indagar en esta cuestión; a ello hay que añadirle el hecho de que el estudio de cómo se comunican los usuarios en contextos digitales ha despertado la atención tanto del público especializado como del que no lo es - por ejemplo, la utilización de emoticonos ha suscitado polémicas controversias sobre su alcance comunicativo-.

Este marcado interés por parte de los lingüistas en investigar los intercambios comunicativos en los contextos digitales se ve reflejado en que este campo de estudio ha sido bautizado de diferentes maneras: comunicación mediada por ordenador (CMO) (Pano, 2008), discurso mediado por ordenador (DMO) (Pano y Moya, 2016), comunicación mediada por computadora (CMC) (Herring y otros, 2013; Cantamutto y Vela Delfa, 2016; Maíz-Arévalo, 2017) o comunicación mediada por tecnología (CMT) (Alcántara-Plá, 2017), entre otras. Dicha diversidad terminológica representa una prueba irrefutable de la relevancia de esta línea de investigación no solamente en el ámbito de la lingüística: también se han publicado trabajos sobre esta cuestión enfocados desde el prisma de la psicología, la antropología, la sociología o la comunicación, por citar tan solo algunas disciplinas.

Sobre la bibliografía anglosajona aseveran Herring y otros (2013) que en la década de los 90 del pasado siglo empiezan a aparecer tímidamente los primeros trabajos sobre comunicación mediada por ordenador. Tales estudios tomaban como objeto de análisis cuestiones relacionadas con la estructura superficial como son los acrónimos, las abreviaturas o los emoticonos. De otra parte, destacan las indagaciones desarrolladas desde el enfoque de la (des)cortesía. En el ámbito hispánico se sigue esta tendencia, si bien se ha de reconocer que las publicaciones son más tardías a la vez que numéricamente inferiores. 
Partiendo de un marco teórico amplio como son los estudios sobre los actos de habla, en general, y los expresivos, en particular, los objetivos de este artículo se centran en analizar - tanto cuantitativa como cualitativamente- los subtipos de actos de habla expresivos utilizados por usuarios con perfiles privados en la red social Facebook. En el corpus analizado se han encontrado diez subtipologías de actos de habla expresivos: felicitar, agradecer, aprobar, animar, admirar, saludar, dar la bienvenida, lamentar, transmitir alegría y desaprobar.

\section{La teoría de los actos de habla: los actos expresivos}

La teoría de los actos de habla — desarrollada a mitad del siglo XX - supuso el nacimiento de la pragmática. El influjo de este postulado también ha afectado al psicoanálisis, la antropología, la sociología o la filosofía (Cuamba Herrejón, 2014: 83). Reyes (1994 [1990]: 15) defiende que esta disciplina ha surgido para encontrar un sentido a la conducta lingüística. Desde su génesis siempre ha estado presente la relación entre semántica y pragmática, la cual es de complementariedad, no de residualidad, en tanto que "la pragmática no es necesaria solamente allí donde la semántica es insuficiente para determinar el significado del hablante: la semántica de por sí2 es siempre insuficiente para eso" (Dascal, 1999: 29). Esta teoría, junto al principio de cooperación de Grice (1957 y 1975) y el principio de la relevancia de Sperber y Wilson (1986), solidifican la pragmática como campo de estudio.

La genialidad de la obra de Austin (1962) — quien es considerado el padre de la pragmática- está motivada por el hecho de romper con la tesis arraigada desde la civilización helénica de que el lenguaje cumple una mera función descriptiva; como señala Rabossi (1999: 54), este filósofo del lenguaje se percata de la discrepancia entre la dimensión ilocucionaria y la dimensión semántica del significado. Con estas palabras da comienzo a la segunda conferencia: "We were to consider, you will remember, some cases and senses (only some, Heaven help us!) in which to say someting is to do something; or in which by saying or in saying something we are doing something" (1962: 12). O, como indica la RAE-ASALE, los enunciados lingüísticos no se limitan a expresar información, sino que "pueden constituir acciones cuando se usan en las circunstancias comunicativas y sociales adecuadas" (2009: 795). Sobre esta base distingue entre los actos de habla locutivos (encargados del sentido literal), los ilocutivos (el emisor indica su intención comunicativa, como puede ser formular una petición) y los perlocutivos (el receptor ha de actuar para que se puedan llevar a cabo, tal es el caso de la persuasión). A su vez, aportó una taxonomía de actos de habla ilocucionarios: judicativos, ejercitativos, compromisorios, comportativos y expositivos.

2 Todas las citas literales incluidas en este artículo respetan las palabras resaltadas en cursiva en sus publicaciones originales. 
Por su parte, Searle (1969, 1975 y 1976), al ser discípulo de Austin (1962), perfecciona el legado sobre los actos de habla de su maestro. La distinción que efectuó Searle (1975) entre actos de habla directos (aquellos en los que hay correspondencia entre significado literal e intención comunicativa) y actos de habla indirectos (aquellos en los que existe divergencia entre significado literal e intención comunicativa) ha sido fuente de obligada referencia para posteriores estudios sobre pragmática. En torno a esta cuestión, defienden Giraldo y Londoño que "el éxito del proceso comunicativo no radica solo en el proceso de decodificiación lingüística de un enunciado, es necesario que el hablante tenga la capacidad de inferir cuál significado quiso dar su interlocutor" (2017: 175). Como reconoce Garrido Medina (1999: 3896), no existe una clasificación de los actos que se pueden efectuar con expresiones en que aparecen los verbos realizativos que haya sido aceptada por unanimidad entre los estudiosos de la materia. La taxonomía que ha desarrollado Searle (1976) sobre los cinco tipos de actos de habla ilocutivos ha gozado de indudable trascendencia, hasta el punto de que el capítulo escrito por Garrido Medina (1999) en la Gramática Descriptiva de la Lengua Española se hace eco de tal clasificación:

1. Actos asertivos: centrados en la verdad o falsedad de los enunciados conforman acciones como afirmar, anunciar o predecir.

2. Actos directivos: su particularidad radica en que el hablante trata de influir en el comportamiento del oyente, como pueden ser las acciones de preguntar, pedir o recomendar.

3. Actos comisivos: el emisor al pronunciar el acto de habla adquiere un compromiso, de tal manera que representan acciones como prometer, ofrecer o jurar.

4. Actos expresivos: el participante transmite puntos de vista, subjetividad o sentimientos con acciones como pedir perdón, felicitar o agradecer.

5. Actos declarativos: esta tipología logra cambiar las ideas del estado de las cosas, por lo que se trata de actos como sentenciar, bautizar o declarar la guerra.

Se han publicado diversos estudios que ofrecen información estadística sobre la frecuencia de uso de estos cinco tipos de actos de habla. Así pues, los datos obtenidos en Vergara Heidke (2006) al analizar editoriales periodísticas señalan un claro predominio de actos asertivos (405), seguidos de actos directivos (131), actos comisivos (2) y actos expresivos (8). Ramayanti y Marlina (2018), tras someter a estudio la película Tangled, hallan los siguientes resultados: el $43.37 \%$ son actos de habla directivos, $24.9 \%$ representativos, $24.4 \%$ expresivos y $7 \%$ comisivos.

Los actos de habla expresivos han recibido menos atención por parte de los investigadores con respecto a otros tipos de actos de habla, como pueden ser los directivos (Maíz-Arévalo, 2017: 152). Ya Austin (1962) se percató de la existencia de actos comportativos, si bien Searle (1976) en su taxonomía contempla los actos expresivos. Este investigador define tales actos 
con estas palabras: "The illocutionary point of this class is to express the psychological state specified in the sincerity condition about a state of affairs specified in the propositional content" (1976: 12). Seguidamente aclara que, cuando se emite un acto de habla expresivo, el hablante no está tratando de hacer que el mundo concuerde con las palabras ni que las palabras coincidan con el mundo, sino que se presupone la verdad de la proposición expresada. Estos subtipos de actos de habla no resultan adecuados para ser utilizados de manera indirecta, dado que su perspectiva ilocucionaria está definida por la expresión del estado psicológico del hablante (Haverkate, 1984: 24).

El propio Searle (1976: 12) cita explícitamente seis verbos expresivos: agradecer, felicitar, pedir disculpas, compadecer, condenar y dar la bienvenida. Enseguida Norrick (1978) aboga por completar esta lista con tres verbos más: Iamentar, perdonar y presumir. En cambio, Carretero y otros (2014) efectúan un análisis de los actos de habla expresivos distinguiendo trece subtipos: disculpa, cumplido, saludo, deseo, agradecimiento, simpatía, preocupación, reproche, directivo, acuerdo, reivindicación, comisivo y tranquilizador.

Otra cuestión teórica sobre los actos de habla expresivos que conviene tener en cuenta es precisamente la división efectuada entre actos de habla expresivos positivos y actos de habla expresivos negativos. Los primeros están conformados por los asentivos (asentir, aceptar, aprobar o aplaudir), los fáticos (saludar, dar la bienvenida o desear lo mejor), los solidarios (felicitar), los exculpatorios (exculpar, perdonar, defender o comprometer) y los compensativos (reconocer o agradecer). En cambio, los actos de habla expresivos negativos incluyen los disentivos (disentir, rechazar, desestimar, desdeñar u oponerse), los inculpatorios (lamentar o disculparse), los incriminatorios y los solidarios (compadecerse, deplorar o comprender) (Puga, 2001: 143).

Desde la perspectiva de la lógica y la inteligencia artificial, Guiraud y otros (2011) proponen un análisis sistemático de los actos de habla expresivos. Tomando como base estos preceptos, Maíz-Arévalo (2017: 162) se hace eco de tal clasificación sobre las emociones que _como en Puga (2001) — se bifurca en dos polos: 1. Positivas; 1.1. Básicas; 1.1.1. Alegría; 1.1.2. Aprobación; 1.2. Complejas; 1.2.1. Celebración; 1.2.2. Admiración; 1.2.3. Satisfacción moral; 1.2.4. Gratitud; 2. Negativas; 2.1. Básicas; 2.1.1. Tristeza; 2.1.2. Desaprobación; 2.2. Complejas; 2.2.1. Culpabilidad; 2.2.2. Arrepentimiento; 2.2.3. Decepción; 2.2.4. Reproche.

\section{Corpus y método}

Para llevar a cabo esta indagación, se analiza el corpus I utilizado en Vivas (2016), el cual está formado por 159 publicaciones —escritas entre 2009 y 2013- que en total suman 867 comentarios extraídos de los perfiles privados de 20 usuarios de la red social Facebook (en concreto, 10 hombres y 10 mujeres) de nacionalidad española, con estudios universitarios y con edades comprendidas entre los 25 y los 35 años. Se ha de precisar que en este corpus las usuarias 
femeninas aportan 91 publicaciones y 428 comentarios, mientras que los usuarios masculinos 68 publicaciones y 439 comentarios; por tanto, existe divergencia en la cantidad de publicaciones, aunque no en comentarios. Para la recopilación de este corpus se ha utilizado el perfil privado de la investigadora con miras a tener acceso a los perfiles privados de otros usuarios; de esta manera, a través de su propio perfil ha podido acceder a las publicaciones, y a los comentarios formulados a raíz de dichas publicaciones, que escribían sus amigos en sus propios muros o bien en los muros de otros usuarios. La selección de este corpus ha estado determinada tanto por el grado de participación en este canal de comunicación como por la predisposición de tales usuarios para permitir recopilar el corpus.

Los temas tratados se circunscriben a cuestiones muy frecuentes en perfiles privados, como son, por ejemplo, las felicitaciones por cumpleaños, dar la enhorabuena por algún logro o comunicar alguna información; en cambio, no aparecen desavenencias que deterioren el vínculo entre los individuos, sino que conforman muestras de manutención y mejora de las relaciones sociales. Las condiciones en que se realiza cada acto de habla aquí analizado quedan establecidas por las normas sociales que rigen los perfiles privados de la red social Facebook y resultan determinantes a la hora de observar la temática de las publicaciones y las respuestas que recibe: las felicitaciones de cumpleaños constituyen un ejemplo muy asiduo en esta red social, igual que dar la enhorabuena a un individuo o bien dar a conocer una determinada información; estas tres tipologías tienen el denominador común de ser actos de habla que favorecen la buena relación entre los sujetos. Se ha de tener presente que cada individuo puede escribir en su propio muro o en los muros de los amigos, elección que ya revela propósitos comunicativos; a la par, los amigos que ven la publicación pueden o no contestar. Aunque con algunas reglas propias de la virtualidad inherente a las redes sociales, la comunicación a través de Facebook se asemeja a la comunicación en presencia entre individuos que se conocen en el mundo real. Cada publicación analizada en este corpus, que cronológicamente fue recopilado entre los años 2009 y 2013, suele centrarse en un único tema siguiendo un orden lineal de comentarios; esto es, no hay metacomentarios, entendiendo por tal un comentario dentro de otro comentario, sino que todos los comentarios nacen del hilo principal que es la publicación.

Ciertamente, las redes sociales facilitan la recogida de corpus de naturaleza digital, frente a otros géneros como pueden ser el correo electrónico, que — por su carácter privadoimplica dificultad para confeccionar un corpus de tal índole (Pano y Moya, 2016: 18). Además, Mancera y Pano (2014: 313) advierten de la necesidad de cavilar sobre el complejo contexto comunicativo que caracteriza las interacciones en redes sociales, como consecuencia de que estos comentarios están insertos en entornos semióticos complejos, como pueden ser los vídeos, las fotografías, las etiquetas o los enlaces, dado que estos elementos a menudo están relacionados con el contenido del mensaje escrito, hasta el punto de que suelen ser vitales para el correcto entendimiento de los mismos. 
En cuanto al método, se ha procedido al estudio cuantitativo y cualitativo de las diferentes subtipologías de actos de habla expresivos hallados en el corpus examinado. En particular, se han encontrado comentarios para agradecer, felicitar y dar la bienvenida (tal como indicaba explícitamente Searle, 1976; el agradecimiento también ha sido estudiado en Carretero y otros, 2014), para lamentar (en sintonía con Norrick, 1978), para saludar (según indican Carretero y otros, 2014), para transmitir alegría, aprobación, admiración y desaprobación (en consonancia con las emociones catalogadas en Maíz-Arévalo, 2017) y para animar. Tras incluir los datos de frecuencia de cada una de estas diez tipologías, se aportan fragmentos que las ilustran.

\section{Análisis y resultados}

Antes de abordar los resultados propios de esta investigación, resulta conveniente conocer información numérica sobre las tipologías de actos de habla hallados en el corpus estudiado. En total se han analizado 867 comentarios, de los cuales 282 (32.52 \%) son actos de habla asertivos, 278 (32.06 \%) conforman actos de habla directivos, 5 (0.57\%) representan actos de habla comisivos y 281 (32.41\%) constituyen actos de habla expresivos; no se ha encontrado ningún caso de acto de habla declarativo y hay 21 (2.42\%) comentarios para los cuales no se ha identificado ningún tipo de acto de habla. Por tanto, los actos de habla asertivos son el grupo más frecuente, seguidos con muy poca diferencia de los actos de habla expresivos y los actos de habla directivos. En cambio, este índice de uso difiere con respecto al estudio de Ramayanti y Marlina (2018), donde los actos de habla expresivos alcanzan el $24.4 \%$, y dista aún más del análisis efectuado por Vergara Heidke (2006), en el cual tan solo se hallan 8 casos en frecuencia absoluta.

Centrados en los actos de habla expresivos existentes en el corpus extraído de la red social Facebook, desde la perspectiva cuantitativa, se observan los siguientes datos de uso _-detallados tanto en frecuencia absoluta como en frecuencia porcentual-:

\section{TABLA 1}

Frecuencia absoluta y porcentual de las subtipologías de actos de habla expresivos

\begin{tabular}{lcclcc} 
SUBTIPOLOGIA & $\begin{array}{c}\text { FRECUENCIA } \\
\text { ABSOLUTA }\end{array}$ & $\begin{array}{c}\text { FRECUENCIA } \\
\%\end{array}$ & SUBTIPOLOGIA & $\begin{array}{c}\text { FRECUENCIA } \\
\text { ABSOLUTA }\end{array}$ & $\begin{array}{c}\text { FRECUENCIA } \\
\%\end{array}$ \\
\hline Felicitar & 127 & 45.19 & Saludar & 19 & 6.76 \\
\hline Agradecer & 47 & 16.72 & Darla bienvenida & 6 & 2.13 \\
\hline Aprobar & 28 & 9.96 & Lamentar & 5 & 1.77 \\
\hline Animar & 24 & 8.54 & Transmitir alegría & 4 & 1.42 \\
\hline Admirar & 20 & 7.11 & Desaprobar & 1 & 0.35 \\
\hline & TOTAL & & & 281 & \\
\hline
\end{tabular}


Como se puede comprobar, felicitar representa la tipología más asidua en el corpus examinado, hasta el punto de sumar el $45.19 \%$ del total (127 comentarios en frecuencia absoluta); esto es, casi la mitad de los actos de habla expresivos son felicitaciones. Dicho predominio se explica por el hecho de que a menudo a través de las redes sociales los usuarios se felicitan principalmente por sus cumpleaños y en otras ocasiones por motivos laborales e incluso personales. A continuación, por orden de uso se hallan: agradecer, con un $16.72 \%$ (47 comentarios en frecuencia absoluta); aprobar, con un $9.96 \%$ (28 comentarios en frecuencia absoluta); animar, con un $8.54 \%$ (24 comentarios en frecuencia absoluta); admirar, con un 7.11 \% (20 comentarios en frecuencia absoluta); y saludar, con un $6.76 \%$ (19 comentarios en frecuencia absoluta). Las otras cuatro subtipologías suponen cifras muy poco representativas: dar la bienvenida asume el $2.13 \%$ (6 comentarios en frecuencia absoluta); lamentar, el 1.77 \% (5 comentarios en frecuencia absoluta); transmitir alegría, el 1.42 \% (4 comentarios en frecuencia absoluta); y desaprobar, el 0.35 \% (1 comentario en frecuencia absoluta).

En cuanto al análisis cualitativo, seguidamente se muestran ejemplos de estas diferentes subtipologías. Como ya se indicaba más arriba, resulta muy habitual encontrar felicitaciones por el cumpleaños (fragmento 1 ) o por otras cuestiones como pueden ser logros laborales (fragmento 2):

[1] muchas felicidades princesica mia!!! hoy tengo doble celebración...es tu cumpleaños y el día q te conocí...es un verdadero honor tenerte en mi vida y por ello me siento muy afortunada..Juanico no podía haber elegido mejor..eres un cielo mi niña..no olvides q te quiero mucho..nos vemos en breve para celebrarlo..un besazo cariño... [perfil 8, publicación 2, comentario 1]

[2] Felicidades muchacha, ya te veo dandole clase el curso que viene a pequeños cabrones... no los mimes demasiado. [perfil 4, publicación 6, comentario 5]

Muy relacionados con la subtipología anterior están los mensajes destinados a dar las gracias por haber sido animado tras conseguir dejar de fumar (comentario 3) o haber sido felicitado por cumplir años (comentario 4):

[3] Gracias por el apoyo a tod@s!! Besos [perfil 8, publicación 6, comentario 5]

[4] CHICOS MUCHÍSMIAS GRACIAS A TODOS, ASÍ ES MUCHO MÁS FÁCIL PASAR A LOS 30!!! UN BESICO MU FUERTE ATOD@S! [perfil 14, publicación 1, comentario 1]

La aprobación en estos contextos comunicativos se puede demostrar de manera muy dispar, ya sea escribiendo la onomatopeya de la risa (ejemplo 5) o bien utilizando el emoticono escrito con signos de puntuación que simboliza un guiño (ejemplo 6):

[5] jejeje ......desde luego [perfil 18, publicación 3, comentario 19] 
[6];-) [perfil 17, publicación 6, comentario 3]

Entre los usuarios de la red social Facebook a veces se intercambian deseos de ánimo, dado que el usuario aludido ha de enfrentarse a una tarea que le resulta tediosa (fragmento 7) 0 por padecer alguna enfermedad (fragmento 8):

[7] Guapa, ànimo!! Mi corazón y todas mis energìas van para ti!!! Un abrazo grande. [perfil 6, publicación 4, comentario 7]

[8] vamos tio. animo. fuerza. te mando mi apoyo. Un abrazo campeón [perfil 14, publicación 2, comentario 9]

En otras ocasiones, las intervenciones están enfocadas a emitir admiración por el usuario aludido, como es alabando la capacidad intelectual (comentario 9) o la belleza física (comentario 10):

[9] eres una cerebrín [perfil 4, publicación 6, comentario 7]

[10] que bonica q eres!! [perfil 10, publicación 5, comentario 1]

Hay intervenciones cuya finalidad se acota a saludar a un usuario, como se manifiesta en estos dos fragmentos que, a su vez, demuestran la relación de confianza existente entre los individuos, si bien en el ejemplo 12 los participantes al no coincidir personalmente utilizan esta red social para mantener el contacto:

[11] Hola morenazaaaaaaa!! [perfil 1, publicación 7, comentario 2]

[12] Hola guapo ke tal estas? haxe muxo ke no se de tí, ni del grupo, aunke observo ke no parais, eso esta bien, con los tiempos ke corre. Besitosssssssssssss muakkkkkkkkkkk [perfil 11, publicación 7, comentario 1]

Otras intervenciones se limitan a dar la bienvenida; este fragmento pertenece a una breve conversación entre amigos en el que uno le da la bienvenida al otro tras viajar a la isla de Fuerteventura:

[13] Bienvenida a casa, wapa!! [perfil 3, publicación 9, comentario 2]

También tienen cabida actos de habla expresivos vinculados con las lamentaciones; la persona que ha publicado el comentario mostrado da a conocer la sensación de hastío que siente ante las modificaciones que le han sido solicitadas en su tesis doctoral:

[14] No puedo estar más hasta los huevos!!!! No se me ocurre volver a hacer una tesis NUNCA MÁS. Tercer envío, después de dos meses de trabajo y..."convendría ampliar algún campo 
más. Te recomiendo estos otros tres libros y un artículo..." Buffffffffffffff. Menos mal que es puente. [perfil 5, publicación 2, comentario 1]

La transmisión de alegría es una subtipología muy poco frecuente en el corpus examinado; en este fragmento se observa que el individuo señala explícitamente su sentimiento de alegría por dicha relación de amistad:

[15] que alegría tenerte como amiga [perfil 7, publicación 4, comentario 1]

Tan solo se ha hallado un comentario de desaprobación, habida cuenta de que dicha emoción negativa no tiene como destinatario a ningún contacto del usuario que lo ha escrito en su muro, sino que habla de la participación en un programa televisivo de un conocido cantante:

[16] Muy mal, Bisbal. [perfil 19, publicación 1, comentario 1]

\section{Conclusiones}

De la presente investigación se pueden extraer estas cinco conclusiones:

1. Los avances tecnológicos se ven reflejados en las nuevas formas de comunicación entre los individuos, y más aún con la generalización de la denominada web 2.0, en la que los usuarios no son meros receptores de información sino que se permite que emitan mensajes. Precisamente el éxito de las redes sociales estriba en dicha cuestión: en que los participantes establecen lazos de comunicación con diferentes individuos. Entre otras disciplinas, la lingüística se ha interesado por este vasto campo de estudio, sobre todo desde el enfoque de la pragmática.

2. Si bien los comienzos de la teoría de los actos de habla se le deben a Austin (1962) y su perfeccionamiento a su discípulo Searle (1969, 1975 y 1976), en la actualidad se ha consolidado como una cuestión de obligada referencia en estudios gramaticales tan sólidos como pueden ser la Gramática Descriptiva de la Lengua Española (1999) o la Nueva gramática de la lengua española (2009). Además, son diversas las investigaciones que indagan en los actos de habla; en esta ocasión, se han examinado los actos de habla expresivos, por lo que se analizan las taxonomías efectuadas por Searle (1967), Norrick, (1978), Carretero y otros (2014) o Maíz-Arévalo (2017).

3. Para estudiar los subtipos de actos de habla expresivos que tienen lugar en la red social Facebook, se ha utilizado el corpus I de Vivas (2016), que está compuesto por 867 comentarios de usuarios con perfiles públicos, de los cuales 281 son actos de habla expresivos. En cuanto al método, con miras a proceder a un análisis tanto cuantitativo como cualitativo, se ha creado una taxonomía ad hoc que combina las necesidades específicas del corpus con las clasificaciones mencionadas más arriba. 
4. Como resultado, se ha de destacar la prevalencia de actos de habla expresivos destinados a felicitar, hasta el punto de que conforman casi la mitad del corpus estudiado. He aquí los subtipos de actos examinados con su respectiva frecuencia de utilización: felicitar (45.19\%), agradecer (16.72\%), aprobar (9.96\%), animar (8.54\%), admirar (7.11\%), saludar (6.76 \%), dar la bienvenida (2.13\%), lamentar (1.77\%), transmitir alegría (1.42\%) y desaprobar $(0.35 \%)$. Dadas las características concretas del corpus - usuarios que se conocen en la vida real cuyas edades están comprendidas entre 25 y 35 años - es normal la relación de cordialidad que presentan, como demuestra el monopolio de actos de habla expresivos sobre emociones positivas.

5. Futuras investigaciones han de arrojar luz sobre los subtipos de actos de habla existentes, así como su frecuencia de utilización en diversos contextos, no únicamente en entornos de comunicación digital. Junto a ello, se ha de observar que la descortesía puede tener cabida en actos de habla aparentemente corteses. Sin ir más lejos, si a priori se puede pensar que los actos de habla expresivos son inherentemente corteses, Alonso Lopera (2013: 92) pone en tela de juicio tal hipótesis, tras analizar si un cumplido puede ser considerado descortés al traspasar la frontera de intimidad del interlocutor. En la misma línea se postulan Hernández Toribio y Deltell Escolar (2017: 204), al indicar que los cumplidos pueden manifestar actos voluntariamente descorteses mediante el uso de crítica, ironía o sarcasmo. En definitiva, resultan necesarias más investigaciones que clarifiquen los complejos mecanismos de comunicación que se dan entre los seres humanos.

\section{Bibliografía citada}

Alcantara-Plá, Manuel, 2017: “El contexto de los mensajes en la comunicación digital” en Mabel Giammatteo, Patricia Gubitosı y Alejandro Parinı (eds.): El español en la red, Madrid / Frankfurt am Main: Iberoamericana/Vervuert, 303-326.

Alonso Lopera, Sergio, 2013: "Reflexiones sobre el uso del acto del habla de los cumplidos", Íkala. Revista de Lenguaje y Cultura 18 (2), 87-93.

Austin, John, 1962: How to do things with words, Cambridge: Harvard University Press [http:// pubman.mpdl.mpg.de/pubman/item/escidoc:2271128:3/component/escidoc:2271430/austin_1962_how-to-do-things-with-words.pdf].

Cantamutto, Lucía, y Cristina Vela Delfa, 2016: "El discurso digital como objeto de estudio: de la descripción de interfaces a la definición de propiedades", Aposta. Revista de Ciencias Sociales 69, 296-323 [http://www.apostadigital.com/revistav3/hemeroteca/cvela2.pdf].

Carretero, Marta, Carmen Maiz-Arévalo y María Ángeles Martínez, 2014: “Hope This Helps!' An analysis of expressive speech acts in online task-oriented interaction by university students" 
en Jesús Romero Trillo (ed.): Yearbook of Corpus Linguistics and Pragmatics 2014, Londres: Springer International Publishing, 261-289.

CRYstal, David, 2001: Language and the Internet, Cambridge: Cambridge University Press.

Cuamba Herrejón, Juan Cruz, 2014: "La apropiación habermasiana y deleuzeana de la teoría de los actos de habla de Austin y Searle", Devenires XV, 83-103.

Dascal, Marcelo, 1999: “La pragmática y las intenciones comunicativas” en Marcelo Dascal (ed.): Filosofía del lenguaje II. Pragmática, Valladolid: Simancas Ediciones, 21-51.

Garrido Medina, Joaquín, 1999: "Los actos de habla: las oraciones imperativas" en Violeta DemonTE e Ignacio Bosque (coords.): Gramática Descriptiva de la Lengua Española. Volumen III, Madrid: Espasa, 3879-3928.

Giammatteo, Mabel, Patricia Gubitosi y Alejandro Parini, 2017: "La comunicación mediada por computadora” en Mabel Giammatteo, Patricia Gubitosi y Alejandro Parinı (eds.): El español en la red, Madrid / Frankfurt am Main: Iberoamericana/Vervuert, 11-26.

GIRALDo, Francisco Luis, y David Alberto Londoño, 2017: "Descripción y referencia: el lenguaje como posibilidad de veracidad. Un asunto de límites de sistemas-mundo y actos de habla”, Cuadernos de Lingüística Hispánica 29, 163-178.

GrICE, Paul, 1957: “Meaning”, Philosophical Review 66 (3), 377-388.

Grice, Paul, 1975: "Logic and conversation" en Peter Cole y Jerry Morgan (eds.): Speech acts (syntax and semantics 3), Nueva York: Academic Press, 41-48.

Guiraud, Nadine, y otros, 2011: "The face of emotions: a logical formalization of expressive speech acts" en Kagan Tumer y otros (eds.): Proceedings of the 1oth International Conference on Autonomous Agents and Multiagent Systems - Volume 3, Richland: International Foundation for Autonomous Agents and Multiagent Systems, 1031-1038 [https://pdfs.semanticscholar.org/c293/7c198ad89688efd07559792c2e5cb273eoga.pdf].

Haverkate, Henk, 1984: Speech acts, speakers and hearers, Ámsterdam/Philadelphia: John Benjamins.

Hernández Toribio, María Isabel, y Luis Deltell Escolar, 2017: "Cumplidos y halagos en el español peninsular: ¿cuestión de términos?”, Onomázein: Revista de Lingüística, Filología y Traducción 37, 188-210 [http://onomazein.letras.uc.cl/Articulos/N37/37_4-Hernandez.pdf]. 
Herring, Susan, Dieter Stein y Tuija ViRTAnen, 2013: "Introduction to the pragmatics of computermediated communication" en Susan HerRIng, Dieter Stein y Tuija VIRTAnen (eds.): Handbook of pragmatics of computer-mediated communication, Berlín: Mouton, 3-31.

Maiz-Arévalo, Carmen, 2017: "Expressive speech acts in educational e-chats", Sociocultural Pragmatics 5 (2), 151-178 [https://www.degruyter.com/downloadpdf/j/soprag.2017.5.issue-2/ soprag-2017-0016/soprag-2017-0016.pdf].

Mancera, Ana, y Ana Pano, 2014: "Las redes sociales como corpus de estudio para el análisis del discurso mediado por ordenador", Janus: Estudios sobre el siglo de oro (Anexo 1: Humanidades Digitales: desafíos, logros y perspectivas de futuro), anexo 1, 305-315.

NorRICK, Neal, 1978: “Expressive illocutionary acts”, Journal of Pragmatics 2 (3), 277-291.

Pano, Ana, y Patricio Moya, 2016: “Una aproximación a los estudios sobre el discurso mediado por ordenador en lengua española”, Tonos Digita/ 30, 1-30 [https://digitum.um.es/xmlui/handle/10201/47991].

PAno, Ana, 2008: Dialogar en La Red. La lengua española en chats, emails, foros y blogs, Berna/ Frankfurt: Peter Lang.

Parrilla, Ernesto Antonio, 2008: "Alteraciones del lenguaje en la era digital”, Comunicar 30, 131136 [https://www.revistacomunicar.com/numeros_anteriores/archivospdf/30/30-21-invesparrilla.pdf].

PugA, Juana, 2001: “Una presentación del software multimedial Actos de Habla”, Revista de Lingüística Teórica y Aplicada 39, 139-153.

Rabossl, Eduardo, 1999: "Actos de habla” en Marcelo Dascal (ed.): Filosofía del lenguaje II. Pragmática, Valladolid: Simancas Ediciones, 53-72.

RAE-ASALE, 2009: Nueva gramática de la lengua española, Madrid: Espasa.

Ramayanti, Dilla, y Leni Marlina, 2018: "The analysis of types illocutionary acts in 'Tangled' movie", E-Journal of English Language \& Literature 7 (1), 27-34.

Reyes, Graciela, 1994 [1990]: La pragmática lingüística. El estudio del uso del lenguaje, segunda edición, Barcelona: Montesinos.

Ridao Rodrigo, Susana, y Francisco José Rodriguez Muñoz, 2013: "Problemas de puntuación en contextos digitales: análisis de comentarios en foros de YouTube", Anuario de Estudios Filo- 
lógicos XXXVI, 83-105 [http://dehesa.unex.es/bitstream/handle/10662/3429/0210-8178_36_83. pdf?sequence=1].

SeArLE, John, 1969: Speech acts: an essay on the philosophy of language, Cambridge: Cambridge University Press.

Searle, John, 1975: "Indirect speech acts" en Peter Cole y Jerry Morgan (eds.): Syntax and semantics. Volume 3, Nueva York: Academic Press, 59-82.

SeARle, John, 1976: "A classification of illocutionary acts", Language in Society 5 (1), 1-23 [https:// sites.duke.edu/conversions/files/2014/09/Searle_Illocutionary-Acts.pdf].

SPerber, Deirdre, y Dan Wilson, 1986: Relevance: communication and cognition, Oxford: Blackwell.

Thurlow, Crispin, 2014: "Disciplining youth: language ideologies and new technologies" en Adam Jaworski y Nikolas Coupland (eds.): The discourse reader, Londres: Routledge, 481-496.

Vergara Heidke, Adrián, 2006: "Actos de habla en editoriales del periódico la Nación de Costa Rica”, Onomázein: Revista de Lingüística, Filología y Traducción 14 (2), 141-161 [http://onomazein.letras.uc.cl/Articulos/14/4_Vergara.pdf].

VIVAS, Julia, 2016: Imagen y (des)cortesía en las redes sociales en español. Un enfoque pragmático. Tesis doctoral, Universidad de Almería.

Yus, Francisco, 2010: Ciberpragmática 2.0. Nuevos usos del lenguaje en internet, Barcelona: Ariel. 